\title{
In search of a new cultural value: The second finding from a bibliometric analysis on biodiversity
}

\author{
Minh-Hoang Nguyen 1,2 \\ Quan-Hoang Vuong 1,3,4
}

1 The Centre for Interdisciplinary Social Research, Phenikaa University, Hanoi 100803, Vietnam

2 Ritsumeikan Asia Pacific University, Beppu, Oita, Japan

3 Centre Emile Bernheim de Recherche Interdisciplinaire en Gestion, Université Libre de Bruxelles, Brussels 1050, Belgium

${ }^{4}$ AISDL, Vuong \& Associates, Hanoi 100000, Vietnam

Working Draft v1

Date: November 8, 2020

No. AISDL-ENV-BIB02

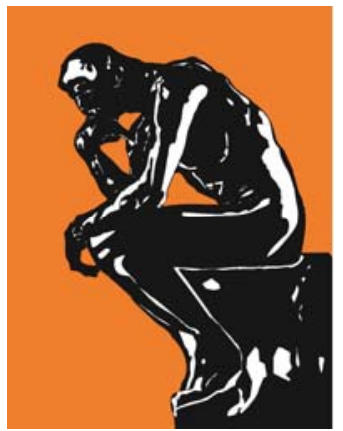

(Un-peer-reviewed draft)

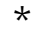




\section{Purpose}

This short piece of communication has the sole purpose of identifying some evidence, supporting our view regarding a possible missing environment-nurturing cultural value [1]. Here, we attempt to examine the presence of cultural studies within the boundary of biodiversity research.

\section{Method}

In what follows, we employ the bibliometric approach that has been presented in Nguyen \& Vuong (2020), including their search queries regarding biodiversity [2]. The number of publications is narrowed down according to the Research Areas appointed by the Web of Science (WoS).

Eventually, the number of cultural studies is acquired. The whole procedure is displayed in Figure 1. The search is conducted on November 8, 2020. It should be noted that we do not limit the search results by language, document type, or publication time when seeking the first collection in the WoS database.

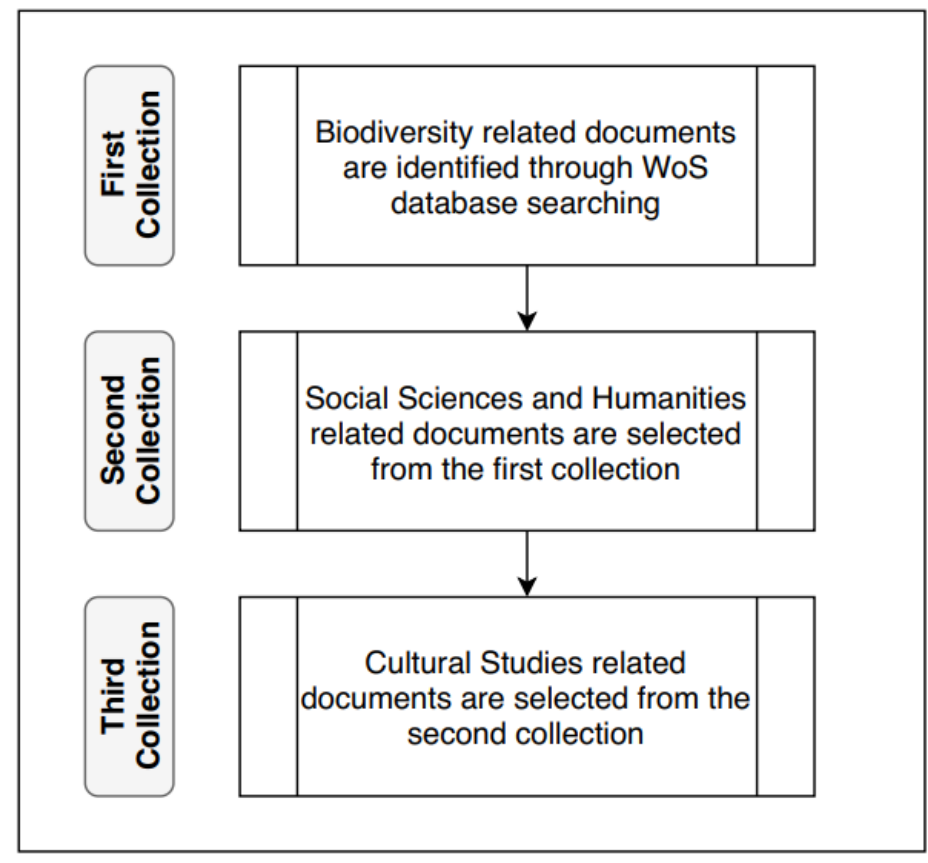

Figure 1: Searching procedure

\section{Findings}

The preliminary result shows that there are 255,298 documents related to biodiversity research. When the scope is narrowed down to Social Sciences and Humanities research related to climate change, we obtain 11,266 records, which account for $4.41 \%$ of the total documents. There remain only 20 documents $(0.007 \%$ of the total publications) when we select solely 'Cultural Studies' research area (see Figure 2). 


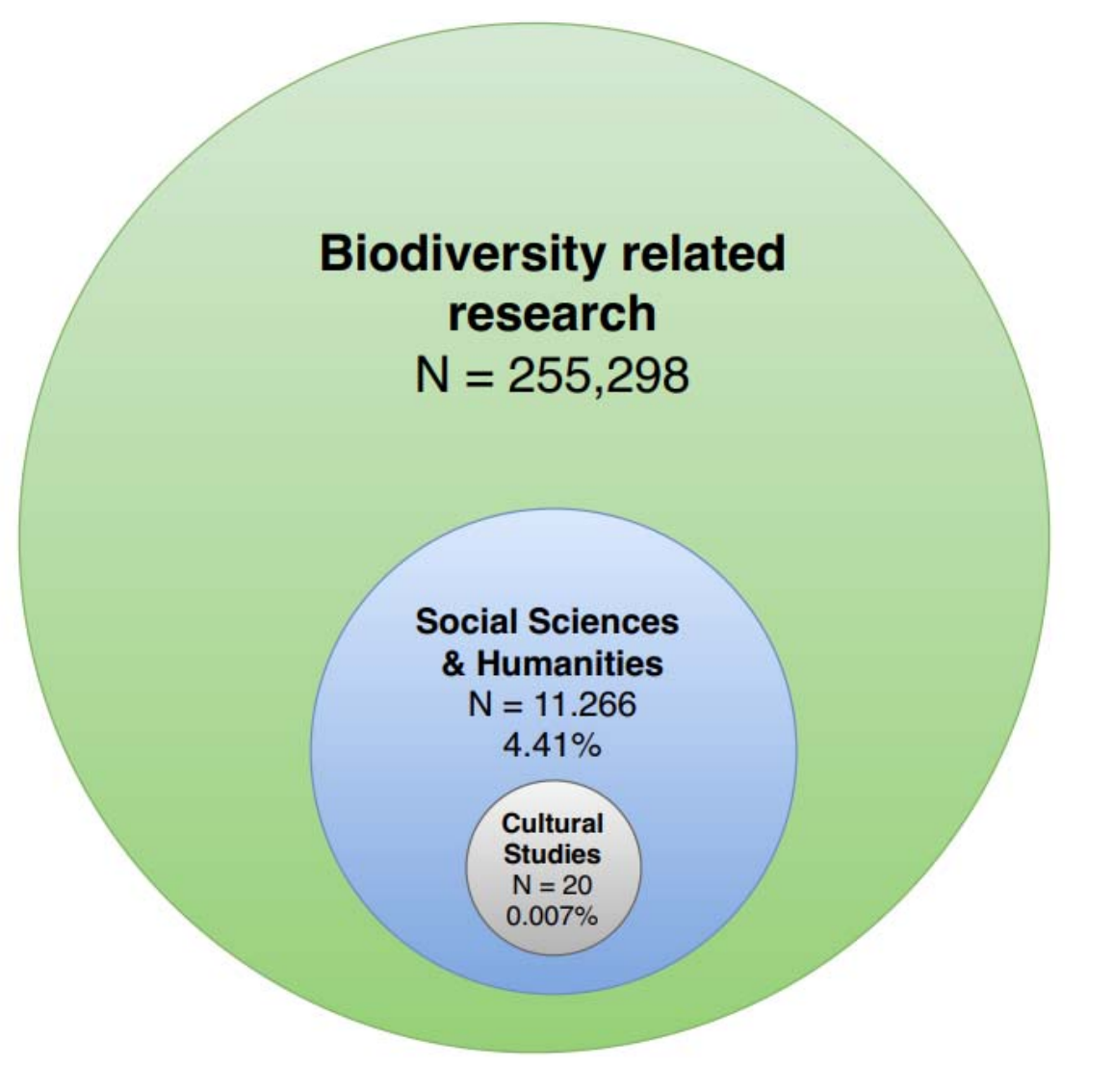

Figure 2: Percentage of Cultural Studies in biodiversity research

Among 20 documents, 16 records are research articles, three records are proceeding papers, and one record is an editorial material. Although the first two studies were conducted in 2004 [3,4], a majority of the documents (90\%) were published after 2009.

\section{Remarks}

To conclude, we observe the existence of cultural studies within the boundary of biodiversity research. Still, its portion, which contributes less than $0.01 \%$ of the total publications, is relatively minute that can be neglected. This finding, together with the former evidence [5], support the argument of Vuong (2020) [1] that current environmental research lacks consideration of cultural issues.

We are aware that this finding is preliminary, and our statement in [1] can potentially be somewhat subversive by nature. Therefore, this communication has no intention of making any conclusion. It rather keeps the finding clean and clear for later use. The effort represents our approach of enabling the mindsponge process to work better and more efficiently in discovering a new paradigm that supports the nurturing of nature and a sustainable environment [6].

\section{References}

[1] Vuong, Q. H. (2020). The semiconducting principle of monetary and environmental values exchange. OSF Preprints. doi:10.31219/osf.io/nv3yz. 
[2] Nguyen, M. H., \& Vuong, Q. H. (2020). Evaluation of the Aichi Biodiversity Targets: The international collaboration trilemma in interdisciplinary research. OSF Preprints. doi: 10.31219/osf.io/84j76.

[3] Barker, J. (2004). The human genome diversity project - 'Peoples', 'populations' and the cultural politics of identification. Cultural Studies 18(4): 571-606. doi: 10.1080/0950238042000232244

[4] Nash, C. (2004). Genetic kinship. Cultural Studies, 18(1), 1-33. doi: 10.1080/0950238042000181593

[5] Nguyen, M. H., \& Vuong, Q. H. (2020). The first finding from a bibliometric analysis concerning a missing environment-nurturing cultural value. OSF Preprints. doi:10.31219/osf.io/bhju8

[6] Vuong, Q. H. (2016). Global mindset as the integration of emerging socio-cultural values through mindsponge processes: A transition economy perspective. In J. Kuada (ed.) Global Mindsets: Exploration and Perspectives (pp. 109-126). London: Routledge. 